EPJ Web of Conferences 109, 05004 (2016)

DOI: 10.1051/epjconf/201610905004

C) Owned by the authors, published by EDP Sciences, 2016

\title{
Statistical Model Analysis of $(n, \alpha)$ Cross Sections for 4.0-6.5 MeV Neutrons
}

\author{
G. Khuukhenkhuu ${ }^{1}$, M. Odsuren ${ }^{1, a}$, Y. M. Gledenov², G. H. Zhang ${ }^{3}$, M. V.Sedysheva ${ }^{2}$, J. \\ Munkhsaikhan $^{1}$, and E. Sansarbayar ${ }^{1,2}$ \\ ${ }^{1}$ School of Engineering and Applied Sciences, Nuclear Research Center, National University of Mongolia, \\ Ulaanbaatar 210646, Mongolia \\ ${ }^{2}$ Frank Laboratory of Neutron Physics, JINR, Dubna, Russia \\ ${ }^{3}$ Institute of Heavy lon Physics, Peking University, Beijing, P.R.China
}

\begin{abstract}
The statistical model based on the Weisskopf-Ewing theory and constant nuclear temperature approximation is used for systematical analysis of the 4.0-6.5 MeV neutron induced $(n, \alpha)$ reaction cross sections. The $\alpha$-clusterization effect was considered in the $(n, \alpha)$ cross sections. A certain dependence of the $(n, \alpha)$ cross sections on the relative neutron excess parameter of the target nuclei was observed. The systematic regularity of the $(n, \alpha)$ cross sections behaviour is useful to estimate the same reaction cross sections for unstable isotopes. The results of our analysis can be used for nuclear astrophysical calculations such as helium burning and possible branching in the $s$-process.
\end{abstract}

\section{Introduction}

The study of $(n, \alpha)$ cross sections is important to calculate an isotope abundance in the Cosmos. For example, the $(n, \alpha)$ reaction can be considered for some isotopes as a possible branch in the s-process. On the other hand, the $(n, \alpha)$ cross section is useful to estimate using the detailed balancing principle the inverse $(n, \alpha)$ reaction cross section of the helium burning process as neutron source in stars [1].

Since 1990 we (collaboration of the Nuclear Research Center, National University of Mongolia, Ulaanbaatar, Mongolia, Institute of Heavy Ion Physics, Peking University, Beijing, P.R.China and the Frank Laboratory of Neutron Physics, Joint Institute for Nuclear Research, Dubna, Russian Federation) have being experimentally investigating the $(n, \alpha)$ reaction in the neutron energy range of several $\mathrm{MeV}$ for broad mass $(6 \leq A \leq 149)$ of target nuclei (see, for example, [2-4]). In addition, a systematical analysis of known fast neutron induced $(n, \alpha)$ reaction cross sections was carried out [5]. A certain regularity of the $(n, \alpha)$ cross sections on the relative neutron excess parameter $(N-Z+0.5) / A$ of the target nuclei for neutron energy range of $\sim 2 \mathrm{MeV}$ [6] and 6-20 MeV [5] was observed. Such a behaviour of the neutron cross sections is termed in literature as the isotopic effect [7]. The statistical model based on the Weisskopf and Ewing theory [8] and constant nuclear temperature approximation [9] was suggested [5] to explain the isotopic effect.

In this work the results of the systematical analysis of our experimental $(n, \alpha)$ cross sections, using the statistical model, for new neutron energy range of 4.0-6.5 MeV are described. Also, $\alpha$ clusterization effect was considered in the $(n, \alpha)$ cross section.

\footnotetext{
a e-mail: odsurenn@gmail.com
} 


\section{The $(n, \alpha)$ Cross Section Formulae}

The direct and pre-equilibrium mechanisms are neglected for several $\mathrm{MeV}$ neutrons and the compound mechanism can be considered, only. Then, according to Bohr's postulate [10], the $(n, \alpha)$ cross section can be written as two stages process:

$$
\sigma(n, \alpha)=\sigma_{c}(n) \cdot G(\alpha)
$$

where:

$$
\sigma_{c}(n)=\pi\left(R+\lambda_{n} / 2 \pi\right)^{2} ; \quad G(\alpha)=\frac{\Gamma_{\alpha}}{\Gamma}=\frac{\Gamma_{\alpha}}{\sum_{i} \Gamma_{i}} .
$$

Here: $\sigma_{c}(n)$ is the compound nucleus formation cross section; where: $R=r_{0} A^{1 / 3}$ is the target nucleus radius; $r_{0}=1.3 \cdot 10^{-13} \mathrm{~cm} ; A$ is the mass number of the target nuclei; $\lambda_{n} / 2 \pi=$ $4.55 \cdot 10^{-13} \mathrm{~cm} / \sqrt{E_{n}(\mathrm{MeV})}$ is the wavelength of the incident neutrons and $E_{n}$ is the incident neutron energy. $\Gamma_{i}, \Gamma_{\alpha}$ and $\Gamma$ are the partial, alpha and total level widths, respectively.

In the framework of Weisskopf-Ewing theory [8], using the constant temperature approximation and the semi-classical formula for the inverse reaction cross section [9] we can get following expression for the $\alpha$-width:

$$
\Gamma_{\alpha}=\frac{2 S_{\alpha}+1}{\pi \hbar^{2}} M_{\alpha} R^{2} \int_{V_{\alpha}}^{E_{\alpha}^{\max }} E_{\alpha}\left(1-\frac{V_{\alpha}}{E_{\alpha}}\right) e^{-\frac{B_{\alpha}+\delta_{\alpha}+E_{\alpha}}{\theta}} d E_{\alpha} .
$$

Here: $S_{\alpha}, M_{\alpha}, E_{\alpha}$ and $V_{\alpha}$ are the spin, mass, energy and the Coulomb potential for the outgoing $\alpha$ particle, respectively; $B_{\alpha}$ is the binding energy of $\alpha$-particle for daughter nucleus; $\delta_{\alpha}$ is the odd-even effect parameter for the Weizsäcker's formula [11]; $\theta=k T$ is the thermodynamic temperature, where $k$ is the Boltzmann constant. Similar formulae can be written for all partial level widths $\Gamma_{i}$.

Then, neglecting the $\gamma$-emission and using the approximation $\Gamma \approx \Gamma_{n}$ for fast neutrons, from (1), (2) and (3) the fast neutron induced $(n, \alpha)$ reaction cross section is determined as follows

$$
\sigma(n, \alpha)=2 \pi\left(R+\lambda_{n} / 2 \pi\right)^{2} e^{\frac{Q_{n \alpha}-V_{\alpha}}{\theta}} .
$$

Similar formula was obtained by Cuzzocrea et al. [12].

The Coulomb potential of $\alpha$-particle can be written [13] in the following form:

$$
V_{\alpha}=2.058 \frac{Z-2}{(A-3)^{1 / 3}+4^{1 / 3}} M e V
$$

The $(n, \alpha)$ reaction energy $Q_{n \alpha}$ can be determined by the Weizsäcker's formula for binding energy. Then, if we neglect the odd-even effect parameter $\Delta=\delta_{f}-\delta_{i}$, from (4) can write the $(n, \alpha)$ cross section as following

$$
\sigma(n, \alpha)=C \pi\left(R+\lambda_{n} / 2 \pi\right)^{2} e^{-K \frac{N-Z+0.5}{A}},
$$

where: $N$ and $Z$ are the neutron and proton number, respectively, for the target nucleus;

$$
\begin{gathered}
C=2 \exp \frac{1}{\theta}\left\{-3 \alpha+\beta\left[A^{2 / 3}-(A-3)^{2 / 3}\right]+\gamma\left(\frac{Z^{2}}{A^{1 / 3}}-\frac{(Z-2)^{2}}{(A-3)^{1 / 3}}\right)+\varepsilon_{\alpha}-V_{\alpha}\right\} \\
K=\frac{2 \xi}{\theta} ; \quad \theta=\sqrt{\frac{U_{a}^{\max }}{a}}=\sqrt{\frac{13.5\left(E_{n}+Q_{n \alpha}\right)}{A-3}}
\end{gathered}
$$


Here: $\alpha, \beta, \xi$ and $\gamma$ are the Weizsäcker's formula constants; and $\varepsilon_{\alpha}=28.2 \mathrm{MeV}$ is the internal binding energy of $\alpha$-particle; $a$ is the level density parameter [14]; $U_{a}^{\max }$ is the maximum excitation energy of residual nucleus;

The parameters $K$ and $C$ in formula (6) can be determined by two methods. First, they can be found by fitting of theoretical cross sections to experimental data as constant parameters at each energy point for all isotopes. Second, $K$ and $C$ parameters can be immediately obtained from the formulae (7) and (8).

\section{Analysis of $(n, \alpha)$ Cross Sections}

\subsection{Systematics of the $(n, \alpha)$ Cross Sections}

The results of the systematics for experimental $(n, \alpha)$ cross sections of the medium mass and heavy nuclei $(40 \leq A \leq 149)$ at neutron energies of $E_{n}=4.0-6.5 \mathrm{MeV}$ by using the formula (6) are shown in Fig. 1.
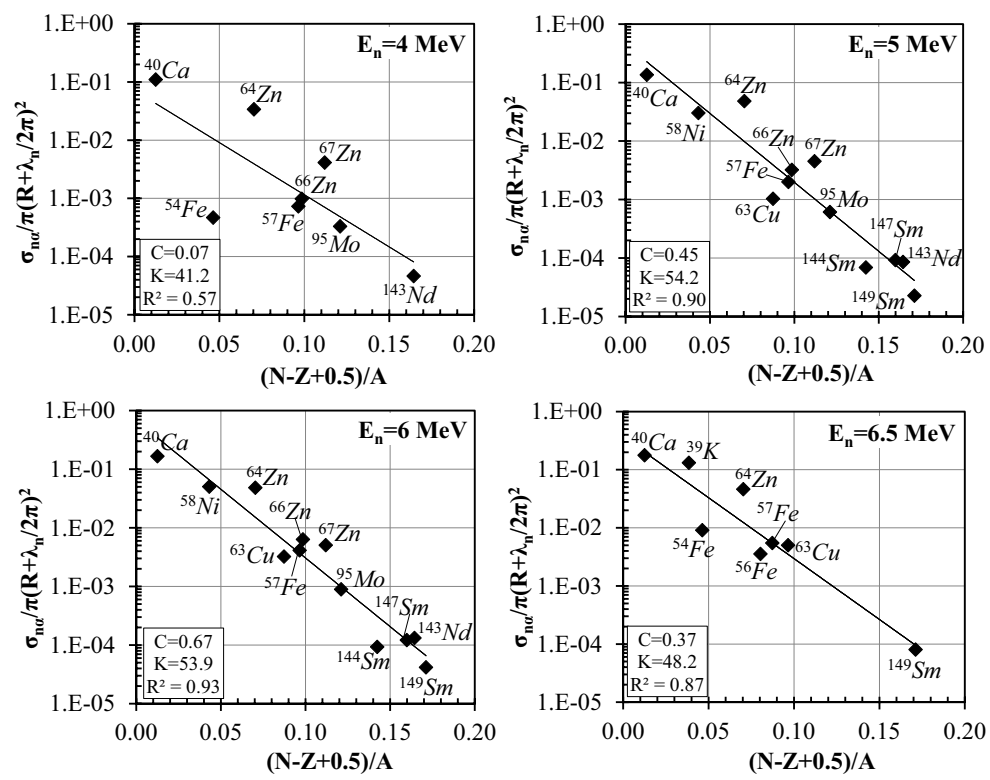

Figure 1. The dependence of the reduced $(n, \alpha)$ cross sections on the neutron excess parameter at $E_{n}=$ 4.0, 5.0, 6.0 and 6.5 MeV.

Similar dependence was obtained in the case of 4.5 and $5.5 \mathrm{MeV}$. The values of fitted parameters $C$ and $K$ are given in Fig.1, also. It is seen that the experimental data of the energy range of 4.0 to 6.5 $\mathrm{MeV}$ is satisfactorily described by the theoretical line drew by formula (6) with the fitted parameters $C$ and $K$. 

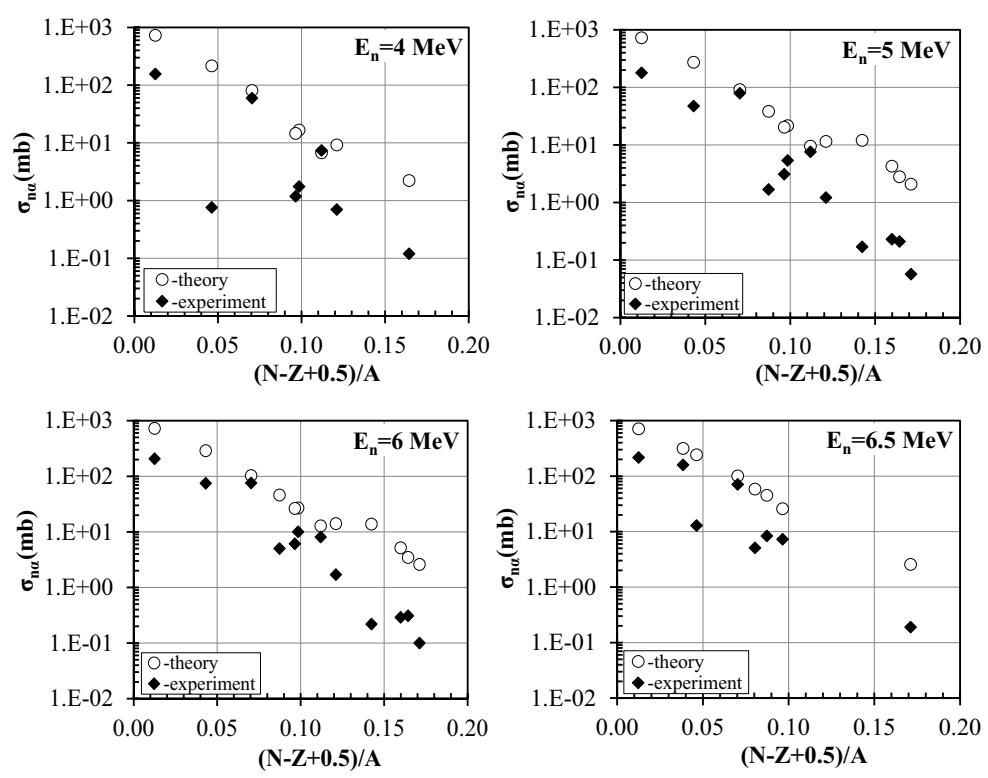

Figure 2. Theoretical and experimental $(n, \alpha)$ cross sections at $E_{n}=4.0,5.0,6.0$ and $6.5 \mathrm{MeV}$.

\subsection{The $(n, \alpha)$ Cross Sections and $\alpha$-clusterization Factor}

The values of the theoretical and experimental $(n, \alpha)$ cross sections for neutron energies of 4.0 to 6.5 $\mathrm{MeV}$ are shown in Fig. 2. The theoretical $(n, \alpha)$ cross sections were calculated by statistical model formulas (6), (7) and (8).

It is seen that the statistical model formulae give overestimated values for the $(n, \alpha)$ cross sections at all neutron energies. We assume that these results, perhaps, are caused by the $\alpha$-particle clusterization effect [15-17].

The $\alpha$-clusterization factor, which takes into account $\alpha$-particle formation probability on the surface of the compound nucleus, was not considered in formulae (6), (7) and (8). So, these formulas should be correct for neutron induced nucleon emission reactions. As to $(n, \alpha)$ reaction, the $\alpha$-clusterization effect should be considered in the cross section formula.

In order to evaluate the $\alpha$-particle formation factor or reduced $\alpha$-width Bethe suggested [18] to use a reduced neutron width: $\gamma_{n}^{2} \approx \gamma_{\alpha}^{2}$

Popov et al. investigated this hypothesis by using the experimental data of the $(n, \alpha)$ reaction for resonance neutrons [15-17] and obtained a following relation for the average reduced neutron-and alpha-widths: $W_{n / \alpha}=\left\langle\gamma_{n}^{2}\right\rangle /\left\langle\gamma_{\alpha}^{2}\right\rangle \approx 2.5-8.0$.

In the case of fast neutron induced $(n, \alpha)$ reaction we suggest to obtain the $\alpha$-clusterization factor by normalization of theoretical cross section to experimental data. This method means that $\alpha$ clusterization factor for $(n, \alpha)$ reaction is determined in comparison with $(n, p)$ reaction assuming the neutron and proton formation factors are the same: $W_{n / p}=\left\langle\gamma_{n}^{2}\right\rangle /\left\langle\gamma_{p}^{2}\right\rangle=1$.

Then, from the normalization of theoretical $(n, \alpha)$ cross section to experimental data the $\alpha$-cluster formation factor was found to be: $W_{p / \alpha}=\left\langle\gamma_{p}^{2}\right\rangle /\left\langle\gamma_{\alpha}^{2}\right\rangle=4.5$. 
Fig. 3 shows that the theoretical $(n, \alpha)$ cross sections calculated by statistical model with the clusterization factor $W_{p / \alpha}=4.5$ are satisfactorily in agreement with experimental data for $E_{n}=4.0-6.5$ $\mathrm{MeV}$. So, taking into account the $\alpha$-clusterization factor, the $(n, \alpha)$ cross section formula (6) can be rewritten in the following form:

$$
\sigma(n, \alpha)=C \pi\left(R+\lambda_{n} / 2 \pi\right)^{2} e^{-K \frac{N-Z+0.5}{A}} \frac{1}{W_{p / \alpha}}
$$
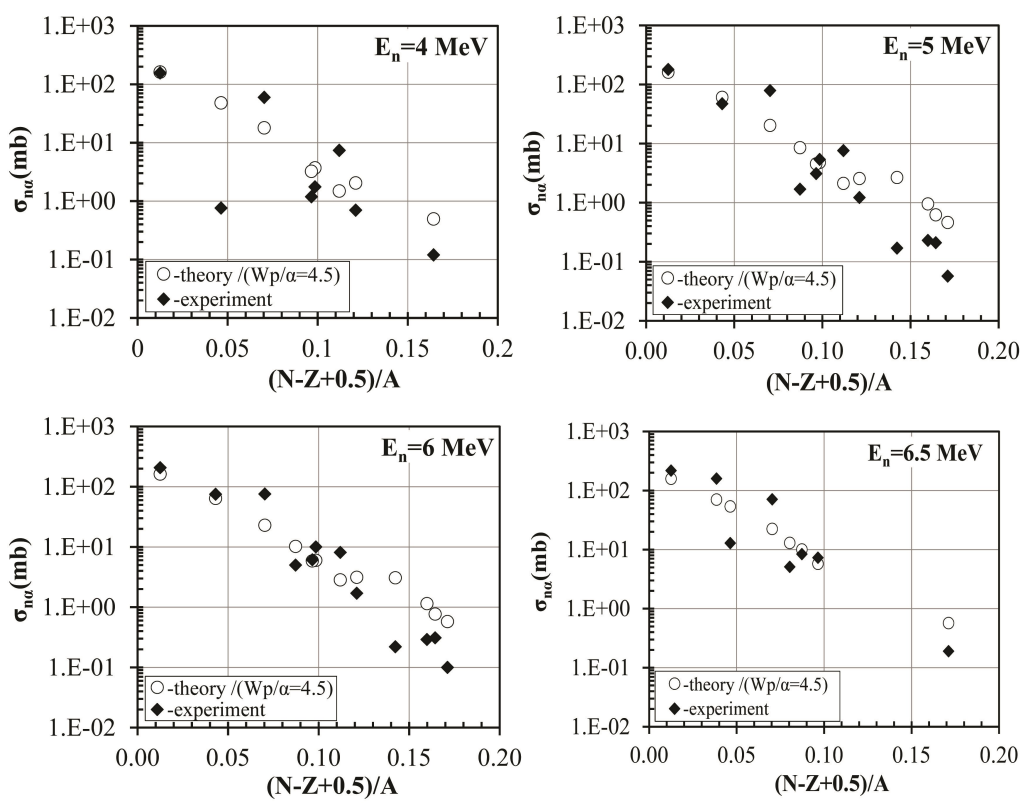

Figure 3. Experimental and theoretical $(n, \alpha)$ cross sections with $\alpha$-cluster formation factor $W_{p / \alpha}=4.5$ for $E_{n}=4.0,5.0,6.0$ and $6.5 \mathrm{MeV}$.

\subsection{Analysis of Existing $(n, \alpha)$ Cross Sections at $E_{n}=6 \mathrm{MeV}$}

Our experimental $(n, \alpha)$ cross sections at $6 \mathrm{MeV}$ together with other existing data [19] are given in Fig. 4.

The Fig. 4 shows that our and other existing $(n, \alpha)$ cross section data at $E_{n}=6 \mathrm{MeV}$ have the same regularity which is described by the statistical model line

\section{Conclusions}

The systematical analysis of $(n, \alpha)$ cross sections for $E_{n}=4.0-6.5 \mathrm{MeV}$ neutrons using the statistical model was carried out. Some systematical regularity of the reduced $(n, \alpha)$ cross sections on the relative neutron excess parameter was observed. The trend of the $(n, \alpha)$ cross sections is satisfactorily described by the statistical model. 


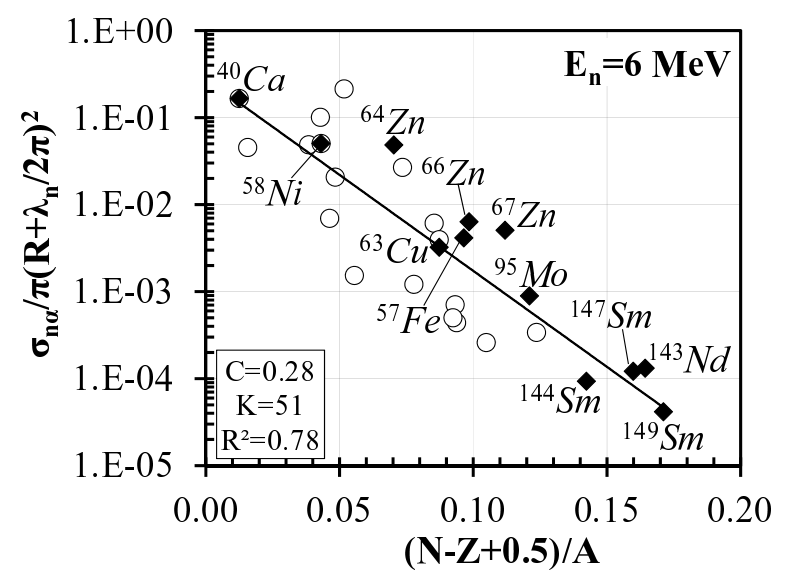

Figure 4. The dependence of our $(\downarrow)$ and other existing $(\bigcirc)$ experimental reduced $(n, \alpha)$ cross sections on the neutron excess parameter.

At the same time, statistical model formulas give overestimated values for the absolute $(n, \alpha)$ cross sections. We assume that these results, perhaps, are caused by the $\alpha$-particle clusterization effect. The clusterization factor was found to be $W_{p / \alpha}=4.5$ for $E_{n}=4.0-6.5 \mathrm{MeV}$ neutrons by normalization of the theoretical $(n, \alpha)$ cross sections to experimental one. This value of the $\alpha$-clustering factor is in good agreement with the result of Popov et al. for resonance neutrons.

Both of our experimental $(n, \alpha)$ cross sections and other existing data at $6 \mathrm{MeV}$ have the same systematical trend which is described by the statistical model, also.

The results of our analysis can be used for nuclear astrophysical calculations such as helium burning and possible branching in the $s$-process.

Systematical regularity of the $(n, \alpha)$ reaction cross sections behaviour in the $\mathrm{MeV}$ energy range of neutrons allows perhaps to estimate the same reaction cross sections for unstable neutron rich and neutron deficient isotopes on which the experimental study of the fast neutron induced reactions is difficult.

\section{Acknowledgements}

This work was financially supported by Mongolian Foundation of Science and Technology.

\section{References}

[1] C.A.Barnes, D.D.Clayton and D.N.Schramm, Essays in Nuclear Astrophysics, (Cambridge University Press, 1982)

[2] G Zhang Guohui et al., Communication of Nuclear Data Progress, China Nuclear Information Centre, 1 (1999)

[3] Jinhua Han et al., Eur. Phys. J. A 51, 12 (2015)

[4] Guohui Zhang et al., Phys. Rev. Lett. 107, 252502 (2011)

[5] G.Khuukhenkhuu et al., Physics of Elementary Particles and Atomic Nuclei, Letters, 11, 749 (2014) 
[6] G.Khuukhenkhuu, M.Odsuren, Yu.M.Gledenov, M.V.Sedysheva and M.Odsuren, Proceedings of the XIX International Seminar on Interaction of Neutrons with Nuclei, ISINN-19, JINR, Dubna, 167 (2012)

[7] H.M.Hoang, U.Garuska, D.Kielan, A.Marcinkowski and B.Zwieglinski, Z. Phys. A 342, 283 (1992)

[8] V.F.Weisskopf and D.H.Ewing, Phys. Rev. 57, 472 (1940)

[9] J.M.Blatt and V.F.Weisskopf, Theoretical Nuclear Physics, (John Wiley and Sons, New York, 1952)

[10] N.Bohr, Nature, 137, 344 (1936)

[11] C.F.Von.Weizsäcker, Zur Theorie der Kernmassen, Z. Phys. A 96, 458 (1935)

[12] P.Cuzzocrea, E.Perillo and S.Notarrigo, Nuovo Cimento.A, 4, 251 (1971)

[13] D.G.Gardner and S.Rosenblum, Nucl. Phys. A 96, 121 (1967)

[14] A.Bohr and B.R.Mottelson, Nuclear Structure, Single Particle Motion, 1, (W. A. Benjamin, Inc. New York, Amsterdam, 1969)

[15] Yu.P.Popov, M.Pshitula, K.G.Rodionov et al., Yadernaya Fizika, 13, 913 (1971)

[16] Yu.P.Popov and W.I.Furman, Lectures of the International School on Neutron Physics, JINR, Dubna, 390 (1978)

[17] N.P.Balabanov, V.A.Vtyurin, Yu.M.Gledenov and Yu.P.Popov, Physics of Particles and Nuclei, 21, 317 (1990)

[18] H.A.Bethe, Nucl. Phys. B, Nuclear Dynamics, Theoretical, Rev. Mod. Phys. 9, 69 (1937)

[19] EXFOR, https://www-nds.iaea.org/exfor/exfor.htm 